\title{
Clinical and experimental applications of the green nanosynthesis for control over the oxidative stress due to heat burn injury
}

\author{
Nikolay Mehandzhiyski ${ }^{1}$, Maria Traykova ${ }^{2}$, \\ Trayko Traykov ${ }^{2}$, Georgeta Bocheva ${ }^{2}$
}

1. University of Forestry, Faculty of Veterinary Medicine;

2. Medical University of Sofia;

\begin{abstract}
Oxidative stress (OS) is defined as imbalance between the accumulation and elimination of free radicals in tissues, in which accumulation prevails. Maintaining homeostatic levels of OS is function of the antioxidant defence (AOD), which is based on catalytic, coordination and irreversible chemical interactions with RS. Burns can be defined as post-traumatic condition accompanied by local and systemic metabolic and structural changes that result in acute inflammation, tissue damage, infections, and even functional impairment of organs distant from the wound. As one of the results from burn injury is increased vascular permeability, pro-inflammatory cytokines and free radicals produced in the wound enter the systemic circulation and may cause systemic inflammatory response, the oxidative stress being a component thereof. The clinical approach to controlling oxidative stress resulting from thermal burns involves antioxidant therapy for patients during the acute phase of the inflammatory response. In animal models and patient studies, administration of antioxidants such as ascorbate, vitamin E, zinc, various antioxidant substances and plant extracts up to 24 hours after the thermal burn has resulted in faster wound healing. Recently, the possibilities of using various plant extracts for control over oxidative stress and treatment of burn wounds have been widely studied. The reason for the widespread use of silver in the treatment of thermal burn wounds is the need to combat antibioticresistant bacterial strains that contaminate the wounds and complicate the condition of the patients. So far there is no evidence of resistance to silver.
\end{abstract}


In general, animal organisms have much better defence from the adverse effect of silver in the wound than cell cultures. Therefore, emphasis is placed on the necessity of studies on animal models that would better simulate the interaction of the human body with silver in the treatment of thermal burn wounds.

Literature analysis gives us grounds to assume that the synthesis of silver nanoparticles from silver nitrate and green tea extract, not in aqueous medium but in glycerin, would result in the formation of a highly bactericidal dispersion system enriched with antioxidants. No one has ever tried such a synthesis of silver nanoparticles for medical applications.

Keywords: Oxidative stress, thermal burns, silver, green nanosynthesis.

\section{Role of oxidative stress in the pathology of burns and in healing of thermal burn wounds}

Oxidative stress (OS) is defined as imbalance between the accumulation and elimination of free radicals in tissues, in which accumulation prevails. This is the first life-threatening response of the living body during change in environment or during internal pathological processes aimed at mobilizing the body during the adaptation or the destruction of harmful internal factors (toxins, viruses, microorganisms, bacteria, degenerated cells of its own tissues). Reactive species (RS) are products of a number of homeostatic metabolic processes. In small amounts, RS act as signalling mediators involved in the oxidation-reduction processes, initiate cell proliferation and differentiation, control the quality of cell cycle and take part in the control of vasomotion. In larger amounts they are part of the immune protection. Their negative effect is manifested as a free radical attack on host healthy cells, resulting in chemical, structural and functional changes at molecular, cellular, tissue, organ, and system levels. This leads to aging, metabolic disorders, diseases and cell death [Traykova M., 2005]. Maintaining homeostatic levels of OS is function of the antioxidant defence (AOD), which is based on catalytic, coordination and irreversible chemical interactions with RS. It is mobilized when the levels of free radicals in the body of the host are elevated and is supressed when these levels drop. Imbalance between the activity of antioxidant defence and the rate of free radical accumulation, regardless of the reason for its occurrence, always leads to new pathological changes in the body and thus to additional complications in the clinical presentation of the disease.

Burns can be defined as post-traumatic condition accompanied by local and systemic metabolic and structural changes that result in acute inflammation, tissue damage, infections, and even functional impairment of organs distant from the wound [Parihar A. et al., 2008]. Burns are extremely complex and involve physiological and metabolic interactions between all important organs and systems. Occurring pathophysiological changes are subject to time dependencies following thermal injury. Burn wounds can be classified into several groups based on injury mechanism: scalding (liquid hot substances), contact burns, chemical, electrical, and radiation burns [Edlich RF. et al., 2016].

The accumulation of toxins and cytotoxic reactive species (RS) in the wound that accompanies the compensatory mechanisms and metabolic responses to the burn also contribute to the local and systemic effects of burns. Despite the major advances in the treatment of burn wounds, the systemic inflammatory response syndrome (SIRS), the sepsis and multiple organ damage are the main causes of disability and 
death in burns [Parihar A. et al., 2008]. In this respect, thermal burn wounds are a typical example [Abdulhalli A. et al., 2014, Bhatia N. et al., 2016].

Wound condition is related to the extent of heat absorption and spread in thermal burns. It depends on thermal conductivity of the affected tissue, which in turn is determined by several factors including [Edlich RF. et al., 2016, Cacir B. and Yegen BC. 2004]: peripheral circulation, water content in the tissue, skin hair, presence or absence of isolating agents (hair and skin sebum). Microcirculation is of most importance for the degree of burns. Thermal burns cause immediate and prolonged inflammatory response that generates toxins and free radicals [Parihar A. et al., 2008, Bhatia N. et al., 2014, Bhatia N. et al., 2016, Cacir B. and Yegen BC. 2004]. As one of the results from burn injury is increased vascular permeability, pro-inflammatory cytokines and free radicals produced in the wound enter the systemic circulation and may cause systemic inflammatory response, the oxidative stress being a component thereof [Edlich RF. et al., 2016]. The interactions between free radicals and radical oxidation products with the components of the antioxidant defence in the body determine whether the inflammatory response will be local and beneficial for repairing tissues in the wound, or it will cause tissue damage, some of which in body organs that are distant from the wound [Parihar A. et al., 2008, Cacir B. and Yegen BC. 2004]. Some of the beneficial features of the reactive oxygen and nitrogen species in wound healing are listed below [Parihar A. et al., 2008, Barbosa E.et al., 2009, Kirn CK. and Roy S. 2008, Shafer M. and Werner S., 2008].

During the phase of infection:

- Reactive oxygen species are mediators of signals that initiate blood coagulation;

- Regulate vasoconstriction and vasodilatation;

- $\mathrm{H}_{2} \mathrm{O}_{2}$ performs fine regulation of the balance between inflammatory control, responsible for the removal of pathogens and the anti-inflammatory control, that prevents the development of too strong inflammatory responses;

- Reactive oxygen and nitrogen species expressed by phagocytes play a central role in protecting the wound from infection;

During the phase of re-epithelisation:

- At low concentrations, reactive oxygen species stimulate the proliferation and migration of epithelial and smooth muscle cells

- $\mathrm{O}_{2}$ converts fibrin into a form that further promotes fibrinolysis

- $\mathrm{H}_{2} \mathrm{O}_{2}$ stimulates epidermal cells growth, which begins 1-2 days after the thermal injury

- Reactive oxygen species stimulate keratinocyte growth factor that is responsible for epidermal regeneration 
During vascularization:

- $\mathrm{H}_{2} \mathrm{O}_{2}$ and the pro-inflammatory response are necessary for wound healing;

- At the same time, control over lipid peroxidation is essential. Evidence for the beneficial role of oxidants during vascularization is the fact that a number of antioxidants can slow it down. As such, some antioxidants are promising as agents to stop vascularization in cancerous tumours;

- NO synthesis plays an important role in the synthesis and accumulation of collagen; strongly affects the synthesis of collagen and the contractile activity of fibroblasts;

- The presence of functionally active iNOS is an essential requirement for normal wound epithelialization.

It has been experimentally proven that small amounts of hydrogen peroxide are present in the wound in the early phase of inflammation (the second day after the injury), that decrease even further at the beginning of new tissue formation ( 5 days after the injury) [Shafer M. and Werner S., 2008]. At the same time, increased levels of superoxide radicals were measured at wound edges. There are experimental data showing significant accumulation of neutrophils in tissues and organs distant from the local thermal burn (gastric mucosa, liver and lungs), accompanied by the formation of large amount of free radicals in them [Parihar A. et al., 2008]. A direct correlation has been observed between the amount of malondialdehyde (MDA) in these organs and the degree of thermal burn. Also, reduction in antioxidant defence in the wound has been observed, indicating an immediate antioxidant response to the free radical processes there. Studies on patients have shown that in severe burns oxidative stress develops in the first five days after the incident, demonstrated by the sharp decrease in antioxidant vitamins and the strong increase in thiobarbituric acid reactive substances (TBARS) [Bertin-Maghit M. et al., 2010].

Based on data in literature, it can be concluded that oxidative stress within certain limits is necessary for the initiation of wound healing processes but its level needs strict control. Control over the level of oxidative stress arising from thermal burns depends on the state of antioxidant defence, in particular on the balance between the bactericidal activity of reactive species released by neutrophils and phagocytes, and the presence and activity of antioxidant defence in the area of the thermal burn. If antioxidant defence is unable to meet the need of eliminating the excess of free radicals in the wound due to increased vascular permeability, these may enter the systemic circulation and, together with pro-inflammatory cytokines, may cause systemic inflammation and systemic oxidative stress. Control over oxidative stress in the wound during the first three days after the thermal injury may significantly help proper wound healing and reduce the risk of oxidative damage in blood plasma and in organs distant from the wound. 


\section{Strategies to enchance antioxidant defence in the treatment of thermal burn wounds}

The clinical approach to controlling oxidative stress resulting from thermal burns involves antioxidant therapy for patients during the acute phase of the inflammatory response [Parihar A. et al., 2008, Barbosa E.et al., 2009, Atiyeh BS. et al., 2007 , Rowan MP. et al., 2015]. In animal models and patient studies, administration of antioxidants such as ascorbate, vitamin E, zinc, various antioxidant substances and plant extracts up to 24 hours after the thermal burn has resulted in faster wound healing. Recently, the possibilities of using various plant extracts for control over oxidative stress and treatment of burn wounds have been widely studied [Rowan MP. et al., 2015, Sarandy MM. et al., 2016, Sapna S. et al., 2016, Mamat AS. et. al., 2016].

At the same time, the level of free radicals can also be reduced by destroying bacteria, fungi and microorganisms that may get into the wound either from an external source or by migration from adjacent skin. In the review by Rowan et al. various approaches to the treatment of burn wounds, used in the practice in recent years are discussed [Rowan MP. et al., 2015].

In the phase of inflammatory response, the gold standard is steroids that reduce inflammation and pain. Inhibitors of prostaglandin synthesis (NSAIDs, glucocorticoids) reduce pain but delay healing. Good results have been obtained with topical opioid administration, but this therapeutic approach requires extensive epidemiological studies to demonstrate efficacy. Oxidative stress in this phase can be controlled by increased levels of antioxidants, free-radical interceptors (vitamin C, vitamin E, melatonin, etc.) as well as infusion of anti-oxidant enzymes (SOD in particular).

During the phase of infection topical antibiotics and silver sulfadiazine (SSD) are most commonly applied. At this stage, antibiotic resistance of bacteria and the invasive fungal infections are particularly challenging. Combating the first requires careful selection of topical antibiotics and combining them according to the dynamics of the infection. Mortality rate is extremely high in patients in which more than $30 \%$ of skin area is affected and which suffer from invasive fungal infections. Sometimes excision of the infected area of the wound is required. The problem of developing resistance to the applied antibiotics still remains very serious.

In many patients, different wound dressings are used. The main criteria they should meet are: to protect against contamination, to prevent from physical damage, to allow gas exchange and to maintain the necessary humidity in the wound. They should be easy to change and should not cause discomfort to the patient.

All of these wound dressings have antibacterial properties due to the silver contained in them. This raises the question about the role of silver in controlling infections in burn wounds. In the care of patients with extensive or deep burn injuries, the antimicrobial efficacy of the wound dressing is a priority. Therefore, the fact that silver-containing dressings may delay healing [Abboud EC. et. al., 2014] is not so significant.

Based on data in literature, it can be assumed that the strategy for controlling oxidative stress in the treatment of burn injuries involves two approaches, which in combination may result in successful treatment of the patient: application of antioxidants and limiting the need of radical-producing immune 
defence against bacteria and microorganisms by destroying them with appropriate medications. In the second approach, ointments, creams and silver containing wound dressings are particularly important.

\section{Role of silver in the treatment of burn wounds}

The reason for the widespread use of silver in the treatment of thermal burn wounds is the need to combat antibiotic-resistant bacterial strains that contaminate the wounds and complicate the condition of the patients. So far there is no evidence of resistance to silver. At present, several silver-based drug formulations are used (Table 3): creams, ointments, solutions, suspensions and wound dressings [Atiyeh BS. et al., 2007, Singh M. et al.,2008, Dai T. et al., 2010, Sharma VK,et al. 2009]. All of these contain silver ions, colloidal silver, or silver nanoparticles.

Table 3: Silver-containing products used in the treatment of thermal burn wounds:

\begin{tabular}{|c|c|c|}
\hline Substance & Product & Manufacturer \\
\hline Ag salts - nitrate, sulphate & $0.5 \%$ solutions & \\
\hline $\begin{array}{l}\text { Charged silver colloidal solutions stabilized by } \\
\text { complexing with low molecular weight proteins }\end{array}$ & $\begin{array}{l}\mathrm{Ag}^{+}, 3-5 \text { ppm, stabilized by } \\
\text { weak electric fields. }\end{array}$ & \\
\hline Ag sulphadiazine (SSD) & Silvadene $^{\mathrm{IM}}$ & \\
\hline Ag sulphadiazine (SSD) & Flammazine $^{\mathrm{TM}}$ & $\begin{array}{c}\text { Smith \& Nephew Healthcare } \\
\text { Limited, Hull, Canada }\end{array}$ \\
\hline Ag sulphadiazine/chlorhexidine & Silverex $^{\mathrm{R}}$ & $\begin{array}{c}\text { Motiff Laboratories Pvt. Ltd. Kare } \\
\text { Health specialties, Verna, Goa }\end{array}$ \\
\hline $\mathrm{SSD} \& \mathrm{CeNO}_{3}$ & Flammacerium $^{R}$ & Solvay, Brussels, Belgium \\
\hline SSD - impregnated lipidocolloid wound dressing & Urgotul SSD ${ }^{R}$ & $\begin{array}{l}\text { Laboratories Urgo, Chenove, } \\
\text { France }\end{array}$ \\
\hline Synthetic net coated with Ag-nanoparticles & Acticoat $^{\mathrm{TM}}$ & $\begin{array}{l}\text { Westaim Biomedical Inc., Fort } \\
\text { Saskatchewan, Alberta, Canada }\end{array}$ \\
\hline Synthetic net coated with Ag-nanoparticles & Silverlon $^{\mathrm{R}}$ & $\begin{array}{l}\text { Argentum Medical, L.L.C., } \\
\text { Lakemont, Georgia }\end{array}$ \\
\hline $\begin{array}{l}\text { Activated charcoal net impregnated with } \mathrm{Ag} \text { - } \\
\text { nanoparticles }\end{array}$ & Actisorb Silver 220 & $\begin{array}{l}\text { Johnson \& Johnson, New } \\
\text { Brunswick, N.J. }\end{array}$ \\
\hline $\begin{array}{c}\text { 70:30 Na:Ag containing carboxymethyl cellulose } \\
\text { hydrofibers }\end{array}$ & Aquacel-Ag hydrofiber & Convatec, Skillman, N.J. \\
\hline $\begin{array}{l}\text { Ag-impregnated polymer film. } \mathrm{Ag} / \mathrm{CaPO}_{4} \text { is a reservoir } \\
\text { for silver ions. }\end{array}$ & Arglaes & Medline, Mundelein, III. \\
\hline $\begin{array}{l}\text { Dense hydrocolloid dressing, in which silver is bound to } \\
\text { the hydrocolloid. }\end{array}$ & Contreet-H & Coloplast, Marietta, Ga. \\
\hline
\end{tabular}

All of these products contain different forms of silver, for which there is evidence that it manifests its bactericidal properties by different mechanisms [Atiyeh BS. et al., 2007, Ribeiro MJ. et al., 2015, Lara HH. 
Et al., 2011, Prabhu S. and Poluose 2012 , You C. et al., 2012, Chen X. et al., 2008, Kim S. et al, 2009, Gunasekaran T. et al., 2012, Martinez-Guiterez F. et al., 2012, Dar A.M. et al., 2013, Lee Y-H. et al., 2014, Han WJ. et al. 2014]. Almost all studies on the cytotoxicity of different silver forms have been carried out in vitro using different cell cultures.

Analysis of the results shows that silver ions and silver nanoparticles manifest their bactericidal properties by various mechanisms. Silver ions react with the thiol groups and disulphide bridges of all biologically active molecules. As these groups are contained in most biologically active proteins, DNA and RNA, their interaction with silver leads to severe changes at all levels of the biological processes in which they are involved. Silver ions are attached to membrane proteins on the surface of viruses, bacteria and microorganisms and damage cell membranes and viral vesicles. Once having entered the cell, these ions are capable of damaging the cell division cycle, take part in oxidation-reduction processes that produce free radicals and disturb signalling mediation. The amounts of glutathione and metallothionein that act as scavengers for $\mathrm{Ag}+$ decrease. There is also rapid drop in the levels of the other radical scavengers engaged in the destruction of the rapidly forming free radicals. Eventually, this leads to elevated levels of oxidative stress. In this article only one aspect of the biological action of silver ions, which is directly related to the generation of free radicals is discussed.

The mechanism of action of silver nanoparticles is different. In vitro toxicological studies have shown that the toxic effect of silver nanoparticles depends not only on their concentration, but also on their size and shape. Considerably higher particle toxicity was observed with diameters about $3 \mathrm{~nm}$ compared to those measuring $25 \mathrm{~nm}$. It has been suggested that silver nanoparticles penetrate the cell through endocytosis or by attaching to the cell wall by binding to the proteins and breaking it through. Once having penetrated the cell, they cluster around membranes (cell membrane, nuclear membrane, vesicles) and the mitochondrial walls, where they form large aggregates. The surface atoms of silver nanoparticles begin to actively participate in electron exchange processes with oxygen molecules turning them into superoxide radicals. The latter, near lipid membranes, initiate chain reactions of free radical superoxidation. The effect of silver nanoparticles occurs later in time than the one of silver ions. At the same time silver nanoparticles are sources of silver ions.

In addition to the advantages in the treatment of burns, the different forms of silver also have disadvantages that need to be taken into consideration when choosing substances and materials for wound treatment. First of all, it should not be forgotten that silver does not recognize host cells and is able of delaying wound healing. Silver salts and solutions are easily oxidized in the air, give unpleasant skin coloration and quickly lose their bactericidal properties. In the wound, they immediately bind to proteins and chloride ions from body fluids and their therapeutic effect is lost. Serum reduces the activity of silver ions in solution about 250 times. This requires that the administration of solutions of silver salts is repeated every 2 hours. Creams, ointments and dressings using SSD have a longer lasting effect, but there is evidence that wound healing is delayed and that some bacteria develop resistance to SSD. Small size of silver nanoparticles make it easier for them to penetrate into circulation and reach various organs in the body (liver, lungs, even brain) where they aggregate. They actively interact with the content of body fluids, damage blood cells, lymphocytes, macrophages. In this way the functions of different organs and systems, distant from the wound, are compromised.

At the same time, however, there are studies showing that the body as a whole can compensate for the harmful effects of silver when it is dosed properly during therapy. All researchers are of the opinion that further and in-depth research is needed on the influence of silver nanoparticles on the levels of oxidative 
stress in the body and the healing of thermal burn wounds. A randomized controlled comparison between the effects of SSD and a cream based on silver nanoparticles has shown that the latter shortens the period of thermal burn wounds healing in humans compared to the classical preparation [Adhya A. et al., 2016].

The pharmaceutical form and the pad that is coated with the silver compounds also have impact on the treatment of thermal burn wounds. In some pads, silver is released in the wound in a controlled manner. In others, bacteria are adsorbed on the pad, where they are killed by the silver nanoparticles. Controlled release of silver is a great advantage because it prolongs the period between changing the patient's dressing. This, on one hand, reduces the possibility of external contamination and, on the other hand, the patient is relieved of the unpleasant sensations of changing the dressing. Creams and ointments are smeared either on gauze or directly on the wound. Their disadvantages are related to the introduction of concomitant substances into the wound that may cause allergic reactions or otherwise affect the condition of the wound.

Literature analysis shows that different forms of silver act by various mechanisms in the treatment of thermal burn wounds. By their pro-oxidant properties, they destroy the biological contaminants in the wound and thereby contribute to reducing the oxidative stress caused by activated neutrophils and phagocytes. This helps the body maintain levels of reactive oxygen and nitrogen species that favour the processes of burn wound healing. Furthermore the risk of oxidative stress development in organs and systems distant from the wound is reduced. If applied in high concentrations, however, silver substances may cause harm by their toxic effect upon the cells in the wound. If they penetrate into blood plasma and/or lymph, silver ions and nanoparticles may cause oxidative stress and tissue damage to organs and systems distant from the wound. The use of different dosage forms and pads improves the efficiency of silver, but the possible adverse effects of the foundation for the creams or wound dressing behaviour should be taken into account.

In general, animal organisms have much better defence from the adverse effect of silver in the wound than cell cultures. Therefore, emphasis is placed on the necessity of studies on animal models that would better simulate the interaction of the human body with silver in the treatment of thermal burn wounds.

\section{The green synthesis of silver nanoparticles as an approach to obtaining bio-safe sanitary products for treatment of thermal burn wounds.}

One of the major problems in the manufacture of silver nanoparticles for medical purposes is the removal of all toxic source substances or synthesis by-products and/or the replacement of the reaction medium with biologically safe one [Prabhu S. and Poluose 2012, Chen X. et al., 2008, Gunasekaran T. et al., 2012, Sharma VK. et al., 2009, Upadhyay LSB. et al., 2015 ]. Particular emphasis is given to "green" synthesis methods where nanoparticles are produced in an environment that does not harm the biological systems. There are several major groups of "green" synthesis methods that rely on the use of synthetic antioxidants, extracts of plant origin, or bacteria for silver nanoparticle synthesis. Molecules that do not alter the metabolic processes in the body or are analogues of products of normal metabolism are used as stabilizers. Polymers that have proven their biological safety are also used. Recently, silver nanoparticles are often being synthesized in plant extract medium with proven antioxidant or bactericidal action. 
Terpenoids, flavones, ketones, amides, aldehydes, polyalcohols, tannins, citrates, carboxylic acids and other phyto-compounds are primarily active during the synthesis of silver nanoparticles, some of which also serving as stabilizers of the suspensions produced.

An easy, inexpensive, and effective way to synthesize silver nanoparticles is the one in green tea extract medium [Nadagouda, M.N. and Varma, R.S. 2008., Moosa A. et al., 2015, Sun Q. et al., 2014, Babu S. et al.,2015]. In these procedures it is not necessary to use high temperature for the synthesis, no special equipment is needed, and the concentration of silver nitrate used is very low (1-10 mM). The resulting nanoparticles are stable. Their bactericidal effect has been demonstrated in cell cultures, but there are no studies on these products in experimental animals.

Glycerine (glycerol) exhibits interesting bactericidal and antiviral properties [Rowe S. et al., 2007, Saegeman VSM. et al., 2008, Stout El. et al., 2012]. When it enters the circulation through the skin, it is absorbed by the enzyme systems in the body without any adverse effects. It is widely used in medicine and cosmetics. It is found to be a slow-acting antiviral, bactericidal and antimicrobial agent. In this respect, its efficacy increases with the concentration and temperature. The optimal temperature for its antimicrobial action is $37^{\circ} \mathrm{C}$ and for the antiviral it is $20^{\circ} \mathrm{C}$. It destroys the bacterial walls in two ways: Accumulating around cell membranes, it reduces the access of unbound extracellular water needed for normal cell functions to cell membranes. In this way cells are gradually dehydrated and either die or go to inactive state. On the other hand, glycerine penetrates cell membranes by the mechanism of facilitated diffusion. It accumulates there, increases osmotic pressure and causes lysis.

Literature analysis gives us grounds to assume that the synthesis of silver nanoparticles from silver nitrate and green tea extract, not in aqueous medium but in glycerin, would result in the formation of a highly bactericidal dispersion system enriched with antioxidants. No one has ever tried such a synthesis of silver nanoparticles for medical applications.

\section{Animal models of thermal burns}

The use of animal models of thermal burns is of great importance for finding new drugs and therapeutic protocols in the treatment of thermal burn injuries. Responses of cell cultures are quite often different from those of animal organisms. The latter have a very wide range of compensatory mechanisms for protection against some undesirable side effects that are unavoidable in cell lines. Mice, hamsters, rats, rabbits, dogs, pigs, sheep are most commonly used as model animals [Abdullahi A. et al., 2016, Mitsunaga JK. et al., 2016]. The skin of each of these animal species has its own similarities and differences with human skin, and this should be taken into account when choosing a model.

The skin of mice has the main layers that are present in human skin (epidermis and dermis) but with significant histological and physiological differences. In addition, mouse coat is thick and hairs have a different growth cycle compared to the one in humans. Mouse skin has a thin skeletal-muscle layer, which is characteristic of only a small section of the human skin at the neck. These considerable differences should be taken into account when looking for analogy in burn wounds healing between mouse and human models. Mouse model of thermal burn provides valuable information about signalling pathways involved in wound healing. The advantage of these experimental animals is low morbidity and good survival in the experiments. The mouse model does not reproduce the healing of human skin, because 
wound closure occurs by contraction rather than epithelization and granulation. In addition, mouse skin has much stronger defence mechanisms against bacterial infection and greater regenerative potential compared to human skin.

The skin of rats has epidermis and dermis, just like human skin, but with the following differences: 1 - Rat skin is much more elastic than human skin; 2 - The close connection between the skin and the subcutaneous structures that is characteristic of human skin is missing; 3 - Rats have the enzyme Lgluconolactone, which converts L-glucono-gama-lactone into vitamin C, while in humans, this enzyme is missing. This is a great advantage for the rat during wound healing, due to the key role of vitamin $C$ for collagen synthesis. The mechanism of wound healing in rats is radically different from that in humans. Their wounds are closed by contraction, not by reepithelization and granulation. Therefore, skin wounds in the rat are healing much faster than in humans. This reduces the risk of infection. The risk of sepsis and immunosuppression in the rat model is much lower than in the case of larger mammals and humans. On the other hand, however, the short healing period makes it possible to track the process more quickly. In general, rats are animals that are easy to grow, they are not fastidious, food is cheap and keeping them does not require large-scale investments. Therefore, the rat model is widely used in the investigation of thermal burn wounds [Mitsunaga JK. et al., 2016, Akscyn R et al., 2015, Cai EJ. et al.,2014, Arbab MH. et al., 2014, Bhatia N. et al., 2014, Shafer M. and Werner S., 2008, Pereira DST. et al., 2012]

Pig skin is closest in structure, morphology and coat to that of humans. The swine model of thermal burn is closest to the processes occurring in human thermal burn wounds. However keeping and growing these animals is very costly and labour intensive. Wound healing does not completely follow the one in humans. Morbidity, risk of infections and sepsis in pigs are much higher than in other animal models.

Each of these models has its place in the research on thermal burn wounds.

We did not find any studies on the dynamics of total antioxidant defence in the development of thermal burn wounds, nor on the effect of silver nanoparticles treatment on this dynamics when reviewing literature on animal models of thermal burn wounds. At the same time, total antioxidant defence is one of the factors that stand between the local oxidative stress in the wound and its spread to other parts of the body. Analysis of the advantages and disadvantages of different animal models has suggested that for the purposes of our study, it is best to choose the rat model where the clinical presentation of burns develops rapidly and there are established standard procedures for modelling the different degrees of thermal burn.

$$
\begin{aligned}
& \text { Abbreviations: } \\
& \text { OC - oxidative stress } \\
& \text { AO - antioxidants } \\
& \text { AOD - antioxidant defense } \\
& \text { AOE - antioxidant enzymes } \\
& \text { CAT - catalase } \\
& \text { GSH - glutathione } \\
& \text { GSPx - glutathione peroxidase } \\
& \text { MDA - malondialdehyde }
\end{aligned}
$$


TBARS - thiobarbituric acid reactive substances.

OS - oxidative stress

RS - reactive species, free radicals

ROS - reactive oxygen species

RON - reactive nitrogen species

SOD - superoxide dismutase

$X$ - xanthine

XO - xanthine oxidase

\section{References}

1. Abboud EC, Legare TB, Settle JC, et al. Do silver-based wound dressings reduce pain? A prospective study and review of the literature. Burns. 2014 (Dec)); 40(1):S40-S47.

2. Abdulhalli A, Amini-Nik S, Jesche MG. Animal models in burn research. Cell Molec Life Sci 2014;71(17):10pages. DOI 10.1007/s00018-014-1612-5, Springer.

3. Abdullahi A, Amini-Nik S, and Jeschke MG. Cardiovascular Dysfunction Following Burn Injury: What We Have Learned from Rat and Mouse Models. Int. J. Mol. Sci. 2016;17(1)53; doi:10.3390/ijms17010053

4. Adhya A, Bain J, Ray $O$, et al. Healing of burn wounds by topical treatment: A randomized controlled comparison between silver sulfadiazine and nanocrystalline silver. J Basic ClinPharmacy 2015 (December-January);6(1): 29-34.

5. Akscyn R, Franklin JL,Gavrikova TA, et al. A rat model of concurrent combined injuries (polytrauma). Int J Clin Exp Med 2015;8(11):20097-20110

6. Arbab MH, Dickey TC, Winebrenner DP, et al. Tetrahertz reflectometry of burn wounds in rat model. Biomed Opt Express 2011;2(8):2339-2347

7. Atiyeh BS, Costagliola M, Shady N. et al. Effect of silver on burn wound infection control and healing: Review of the literature. Burns 2007; 33:139-148

8. Babu S, Claville MO, Ghebreyessus K. Rapid synthesis of highly stable silver nanoparticles and its application for colorimetric sensing of cysteine. J exp Nanosci 2015;10(16):1242-1255.

9. Barbosa E, Faintuch J, Machado M, et al. Supplementation of Vitamin E, Vitamin C, and Zinc Attenuates Oxidative Stress in Burned Children: A Randomized, Double-Blind, Placebo-Controlled Pilot Study. J Burn Care 2009; 30(5):859-866

10. Bertin-Maghit M, Goudable J, E. Dalmas E, et al. Time course of oxidative stress after major burns. Intensive Care Medicine 2000 (June); 26( 6): 800-803

11. Bhatia N, Kaur G, Soni et al.mEvaluation of the wound healing potential of isoquercetin-based cream on scald burn injury in rats. Burns \& Trauma 2016;4:7; DOI: 10.1186/s41038-016 -0032-1

12. Bhatia N, Singh A, Scharma R, et al. Evaluation of burn wound healing potential of aqueous extract of Morus alba based cream in rats. J Phytopharmacology 2014;3((6):378-383

13. 13. Cacir B, Yegen BC. Systemic responses to burn injury. Turk. J. Med. Sci. 2004;34: 215-226.

14. 14. Cai EJ, Ang $\mathrm{CH}$, Raju A., et al. Effects of hyperbaric oxygen therapy on experimental burn wound healing in rats: A randomized controlled study. UHM 2005;32(1):1-9

15. 15. Cai EJ, Ang CH, Raju A., et al. Creation of consistent burn wounds: Rat model. Archives of Plastic Surgery 2014;41:317-324

16. Chen X, Schluesener HJ. Nanosilver: A nanoproducy in medical application. Toxicol Lett 2008; 176:1-12

17. Dai $T$, Huang $Y-Y$, Sharma SK. et al. Topical antimicrobials for burn wound infections. Res Pat Antiinfect Drug Discov 2010(June);5(2):124-151 
18. Dar A.M, Ingle A, Rai M. Enhanced antimicrobial activity of silver nanoparticles synthesized by Cryphonectria sp. Evaluated singly and in combination with antibiotics. Nanomedicine: Nanotech Biol Med 2013;9:105-110

19. Das U, Behera SS, Singh S, et al. Progress in the Development and Applicability of Potential Medicinal Plant Extract-Conjugated Polymeric Constructs for Wound Healing and Tissue Regeneration. hytother. Res. 2016; 30: 1895-1904. doi: 10.1002/ptr.5700.

20. Edlich RF, Drake DB, Long WB III. Thermal Burns. MedScape, Drugs\&Diseases 2016; Seen December 10-th at http://emedicine.medscape.com/article/1278244-overview.

21. Gunasekaran T, Nigusse T, Dhanaraju DD. Silver nanoparticles as real topical bullets for wound healing. J Am College of Clinical Wound Specialists 2012; 3:82-96

22. Han WJ, Gurunathan S, Jeong J-K, Choi Y-J, et al. Oxidative stress mediated cytotoxicity of biologically synthesized silver nanoparticles in human lung epithelial adenocarcinoma cell line. Nanoscale Res Lett 2014;9(1):459-475. http:://wwwnanoscalelett.com/content/9/1/459

23. Jackson DM. The diagnosis of death of burning. Br J Surg 1953(May);40(164):588-596 [Medilne]

24. Kim S, Choi JE, Choi J, et al. Oxidative stress-dependent toxicity of silver nanoparticles in human hepatoma cells. Toxicology in vitro 2009;23:1076-1084.

25. Kirn CK, Roy S. Redox signals in wound healing. Biochimica et Biophysica acta 2008;1780:13481361.

26. Lara HH, Garza-Trevino EN, Ixtepan-Turrent L, et al.. Silver nanoparticles are broad-spectrum bactericidal and virucidal compounds. J Nanobiotechnology 2011;9:30 http://wwwinanobiotechnology.com/content/9/1/30

27. Lee $\mathrm{Y}-\mathrm{H}$, Cheng F-Yu, Chiu H-W, et al. Cytotoxicity, oxidative stress, apoptosis and autophagic effects of silver nanoparticles in mouse embryonic fibroblasts. Biomaterials 2014;35:4700-4715

28. Mamat AS, Ahmad MZ, Salam F. An Update Review on Polyherbal Formulation: A Global Perspective. Systematic Reviews in Pharmacy 2016;7(1):35-41

29. Martinez-Guiterez F, Thi EP, Silverman JM, et al. Antibacterial activity, inflammatory response, coagulation and cytotoxicity effects of silver nanoparticles. Nanomedicine: Nanotechnol Biol Med 2012;8:328-336

30. Mitsunaga JK, Gragnani A, Ramos MLC, et al. Rat experimental model for burns. A systematic Review. Acta Cirurgica Brazileira 2012;27(6):8 pages; thhp://dx.doi.org/10.1590/ S010286502012000600010

31. Moosa A, Ridha AM, Allawi MH. Green Synthesis of Silver Nanoparticles using Spent Tea Leaves Extract with Atomic Force Microscopy. International Journal of Current Engineering and Technology 2015;5(5):3233-3241

32. Nadagouda, M.N. , Varma, R.S. Green synthesis of silver and palladium nanoparticles at room temperature using coffee and tea extract. Green Chemistry 2008;10(8), p.859-861

33. Parihar A, Parihar MS, Milner S, et al. Oxidative stress and antioxidative mobilization in burn injury. Burns 2008; 34: 6-17.

34. Pereira DST, Lima-Ribeiro MHM, de Pontes-Filho NT, et al. . Development of Animal Model for Studying Deep Second-Degree Thermal Burns. Journal of Biomedicine and Biotechnology 2012 (2012); Article ID 460841, 7 pages; http://dx.doi.org/10.1155/2012/460841

35. Prabhu S, Poluose EK. Silver nanoparticles: mechanism of antimicrobial action, synthesis, medical applications and toxicity effects. International Nano Letters 2012; 2:32-42. http://www.inljournal.com/content/2/1/32

36. Ribeiro MJ, Maria VL, Scott-Fordsmand J.J, et al. Oxidative stress mechanisms caused by Ag Nanoparticles (NM\#))K) are different from those of AgNO3: Effects in the soil invertebrate Enchytraeus crypticus. Int. J. Res. Public Health 2015;12:9589-9602.

37. Rowan MP, Cancio LC, Elster LCA, B et al. . Burn wound healing and treatment: review and advancements. Crit Care. 2015; 19: 243.

38. Rowe S, Himi S, Wood F. The use of ichthamol glycerin in burn wound care: a review. Primaty Intent 2007;15(1):29-32 
39. Saegeman VSM, Ectors NL, Lismont D, et al. Short- and long-term bacterial inhibiting effect of high concentrations of glycerol used in the preservation of skin allografts. Burns 2008;34: 205211

40. Sapna S, Anju D; Sanju N. Traditional Indian medicinal plants with potential wound healing activity: a review. International Journal of Pharmaceutical Sciences and Research 2016(May); 7(5): 1809-1819.

41. Sarandy MM, Lopes FB, Pinto da Matta SL, et al. Effect of Topical Administration of Fractions and Isolated Molecules from Plant Extracts on Skin Wound Healing: A Systematic Review of Murine Experimental Models. Mediators of Inflammation (2016); (Volume 2016) Article ID 4916068, 25 pageshttp://dx.doi.org/10.1155/2016/4916068

42. Shafer M, Werner S. Oxidative stress in normal and impaired wound repair. Pharmacological Res. 2008;58:165-171

43. Sharma VK, Yngard RA, Lin Y. Silver nanoparticles: Green synthesis and their antimicrobial activities. Adv Coll Interf Sci 2009; 145:83-96.

44. Singh M, Singh S, Prasad S, Gambhir IS. Nanotechnology in medicine and antibacterial effect of silver nanoparticles. DIGEST j Nanomater Biomolecules 2008;3(3):115-122

45. Stout El, McKessor A. Glycerin-based hydrogel for infection control. Adv Wound Care 2012;1(1):48-51

46. Sun Q, CaiX, Li J, Zheng M, et al.. Green synthesis of silver nanoparticles using tea leaf extract and evaluation of their stability antibacterial activity. Colloids and Surface A: Physicochemical and engineering aspects 2014;444:226-231

47. Traykova M, Kostova I. Coumarins and Oxidative Stress. Int. J. Pharmacol. 2005; 1(1):29-32

48. Upadhyay LSB, Verma N. Recent developments and applications of plant extract-mediated synthesis of silver nanoparticles. Analytical Letters 2015;48(17):2676-2692.

49. Yao L, Li Z-R, Su W-R. et al. Role of mesenchymal stem cells on cornea wound healing induced by acute alkali burn. PLOS one 2012(February);7(2):e30842, 7 pages. doi:10.1371/journal.pone0030842

50. You C, Han C, Wang X, et al. The progress of silver nanoparticles in the antibacterial mechanism, clinical application and cytotoxicity. <ol Biol Rep 2012; 39:9193-9201

\section{Corresponding author:}

Nikolay Mehandzhiyski

10 Kliment Ohridski Blvd,

1000 Sofia, Bulgaria

email: poseidon77@abv.bg 